\title{
Platinum-Catalyzed Hydrative Cyclization of 1,6-Diynes for the Synthesis of 3,5-Substituted Conjugated Cyclohexenones
}

\section{Chen Zhang ${ }^{1}$, Jian-Feng Qi ${ }^{1}$, Dong-Mei Cui ${ }^{2, *}$, Qian Wang ${ }^{2}$ and Xiu-Li Wang ${ }^{3}$}

1 School of Pharmaceutical Sciences, Zhejiang University, Hangzhou 310058, China

2 College of Pharmaceutical Science, Zhejiang University of Technology, Hangzhou 310014, China

3 College of Chemistry and Bioengineering, Guilin University of Technology, Guangxi, Guilin, China

* Author to whom correspondence should be addressed; E-Mail: cuidongmei@ zjut.edu.cn;

Tel.: +86-571-88320320; Fax: +86-571-88320320.

Received: 4 June 2010; in revised form: 24 June 2010 / Accepted: 5 July 2010 /

Published: 23 July 2010

\begin{abstract}
We have developed a $\mathrm{Pt}(\mathrm{COD}) \mathrm{Cl}_{2}$-catalyzed hydrative cyclization of 1,6-diynes leading to the formation of functionalized cyclohexenones in good yields.
\end{abstract}

Keywords: diyne; catalysis; hydrative cyclization; cyclohexenones

\section{Introduction}

Cyclohexenone derivatives are not only key intermediates in organic synthesis, but they also exhibit important pharmacological activities [1-4]. Extensive synthetic efforts for conjugate cyclohexenones have been reported, in which an annulation approach from acyclic precursors constitutes a useful entry [5-13]. Despite this advance, there is still a great need to develop more convenient catalytic systems that can accommodate such attractive features as easily accessible starting materials, mild reaction conditions, and absence of co-products. The newly developed metal-catalyzed hydrative cyclization reaction is not only an especially attractive "green" procedure, but also an ideal synthetic method for preparing cyclic enone compounds [14-20]. The reported examples include hydrative cyclization of 1,n-diynes [14-17], 1-yne-5-enones [18], 1-en-5-ynes [19] and diynnols [20]. Recently, Liu and coworkers reported a $\mathrm{PtCl}_{2}$-catalyzed hydrative cyclization of internal triynes to yield bicyclic spiroketones [21-23]. As part of our ongoing studies on metal-catalyzed atom-economical reactions, 
we succeeded in synthesizing conjugate cyclohexenone ring systems using the hydrative cyclization of 1,6-diynes with $\left(\mathrm{PPh}_{3}\right) \mathrm{AuMe}$ as a catalyst [24,25]. Herein, we report our studies on the use of $\mathrm{Pt}(\mathrm{COD}) \mathrm{Cl}_{2}$ as a catalyst in this cyclization.

\section{Results and Discussion}

Initial hydrative cyclization experiments of 1,6-diyne $1 \mathbf{a}(0.5 \mathrm{mmol})$ with $\mathrm{H}_{2} \mathrm{O}(0.5 \mathrm{mmol})$ at $70{ }^{\circ} \mathrm{C}$ for $4 \mathrm{~h}$ in a sealed-tube were performed to screen catalysts. $\mathrm{Pt}(\mathrm{COD}) \mathrm{Cl}_{2}\left(\mathrm{COD}=\right.$ cyclooctadiene) $^{2}$ combined with methanesulfonic acid $\left(\mathrm{CH}_{3} \mathrm{SO}_{3} \mathrm{H}\right)$ showed good catalytic activity in this reaction, furnishing cyclohexenone $\mathbf{2 a}$ in $75 \%$ yield without the formation of the corresponding hydration or methanol adducts (Table 1, entry 1), while the reaction conducted in the absence of $\mathrm{CH}_{3} \mathrm{SO}_{3} \mathrm{H}$ did not yield the cyclic product (Table 1 , entry 2 ). Trifluromethanesulfonic acid $\left(\mathrm{CF}_{3} \mathrm{SO}_{3} \mathrm{H}\right)$ can also serve as an excellent co-catalyst. $\mathrm{PtCl}_{2}$ in combination with $\mathrm{PPh}_{3}$ gave 2a in lower yield (Table 1, entry 4). There was no reaction with other homogeneous metal complex systems, such as $\operatorname{Pd}\left(\mathrm{P}^{i} \mathrm{Pr}_{3}\right)_{2} \mathrm{Cl}_{2}$ and $\mathrm{Ru}(\mathrm{COD}) \mathrm{Cl}_{2}$ (Table 1, entries 5 and 6). During further optimization of the reaction conditions, we found that a lower catalyst loading ( $2 \mathrm{~mol} \%$ ) afforded the product with decreased yield (Table 1, entry 3 ).

Table 1. Pt(II)-Catalyzed synthesis of cyclohexenone from 1,6-diyne ${ }^{a}$.

\begin{tabular}{|c|c|c|c|c|}
\hline & $\mathrm{MeO}_{2} \mathrm{CeO}{ }_{1 \mathrm{a}} \mathrm{C} \sqrt{\equiv}+1$ & $\frac{\text { cat., } 70^{\circ} \mathrm{C}}{\mathrm{MeOH}}$ & ${ }_{2 a}^{\mathrm{O}_{2} \mathrm{C}}$ & \\
\hline Entry & Catalyst (mol\%) & $\begin{array}{c}\mathrm{CH}_{3} \mathrm{SO}_{3} \mathrm{H} \\
(\mathrm{mol} \%)\end{array}$ & $\begin{array}{l}\text { Time } \\
\text { (h) }\end{array}$ & $\begin{array}{c}\text { Yield }^{b} \\
(\%)\end{array}$ \\
\hline 1 & $\mathrm{Pt}(\mathrm{COD}) \mathrm{Cl}_{2}(5)$ & 50 & 3 & 75 \\
\hline 2 & $\mathrm{Pt}(\mathrm{COD}) \mathrm{Cl}_{2}(5)$ & - & 3 & Trace \\
\hline 3 & $\mathrm{Pt}(\mathrm{COD}) \mathrm{Cl}_{2}(2)$ & 50 & 3 & 21 \\
\hline 4 & $\mathrm{Pt}\left(\mathrm{PPh}_{3}\right) \mathrm{Cl}_{2}(2)$ & 50 & 4 & 12 \\
\hline 5 & $\mathrm{Pd}\left(\mathrm{P}^{i} \operatorname{Pr}_{3}\right) \mathrm{Cl}_{2}(2)$ & 50 & 4 & NR \\
\hline 6 & $\mathrm{Pt}(\mathrm{COD}) \mathrm{Cl}_{2}(2)$ & 50 & 4 & $\mathrm{Nr}$ \\
\hline
\end{tabular}

${ }^{\mathrm{a}}$ The reactions were performed with $1 \mathrm{a}(0.5 \mathrm{mmol}), \mathrm{H}_{2} \mathrm{O}(0.5 \mathrm{mmol}), \mathrm{CH} 3 \mathrm{SO} 3 \mathrm{H}(1-50$ $\mathrm{mol} \%)$, and catalyst $(2-5 \mathrm{~mol} \%)$ in $\mathrm{MeOH}(2 \mathrm{~mL})$ at $70^{\circ} \mathrm{C} .{ }^{\mathrm{b}}$ Isolated yields.

In order to demonstrate the efficiency and scope of the present method, we applied the optimum conditions of entry 1 in Table 1 to the hydrative cyclization of several 1,6-diyne substrates bearing a variety of functionalities at their 4-positions. The results are summarized in Table 2. Terminal malonate derivatives $\mathbf{1 a}$ and $\mathbf{1 b}$ were found to be good substrates (Table 2, entries 1 and 2). This is quite similar to the results of $\mathrm{Au}$ (I)-catalyzed reactions [24,25]. To our delight, the presence of two hydroxyl groups as in compound 1c was tolerated, thus providing cyclohexenone $\mathbf{2 c}$ bearing hydroxyl groups with no intramolecular alcohol addition products (Table 2, entry 3) [26,27]. Protecting groups such as the single methyl ether in $\mathbf{1 d}$ or the double methyl ether in 1e were also compatible with the present method (Table 2, entries 4 and 5). Cyclic products with different substituent group pairs, such as the diphenylphosphoryl and ethoxycarbonyl in $\mathbf{2} \mathbf{f}$, or the phenyl and methoxycarbonyl in $\mathbf{2 h}$, were also obtained in good yields (Table 2, entries 6 and 8). The acetylacetone derivative $\mathbf{1 i}$ and its reduced 
derivative $\mathbf{1 j}$ were transformed into cyclic products $\mathbf{2} \mathbf{i}$ and $\mathbf{2} \mathbf{j}$ (Table 2, entries 9 and 10). In our hands the spirocyclic compound $\mathbf{2 k}$ bearing a fluorene moiety was successfully obtained from diyne $\mathbf{1 k}$ in $48 \%$ yield (Table 2, entry 11 ).

Table 2. Pt (II) catalyze hydrative cyclyzation reaction of 1, 6-heptadiynes a .

\begin{tabular}{|c|c|c|c|c|c|c|}
\hline entry & 1,6-diynes & 1 & time (h) & product & 2 & yield $(\%)^{b}$ \\
\hline 1 & ${ }_{2}{ }_{2}^{C} \times=$ & $1 a$ & 3 & & $2 a$ & 75 \\
\hline 2 & $\overline{\overline{ }}$ & $1 b$ & 3 & & $2 b$ & 88 \\
\hline 3 & $=$ & 1c & 4.5 & & 2c & 39 \\
\hline 4 & & 1d & 6 & & $2 d$ & 61 \\
\hline 5 & $\overline{\overline{ }}$ & $1 e$ & 12 & & $2 e$ & 65 \\
\hline 6 & & $1 f$ & 6 & & $2 f$ & 78 \\
\hline 7 & & $1 \mathrm{~g}$ & 3 & & $2 g$ & 75 \\
\hline 8 & & $1 \mathrm{~h}$ & 3 & & $2 h$ & 69 \\
\hline 9 & & $1 \mathrm{i}$ & 5 & & $2 \mathbf{i}$ & 62 \\
\hline 10 & & $1 \mathrm{j}$ & 5 & & $2 j$ & 47 \\
\hline $11^{\mathrm{c}}$ & & $1 k$ & 13 & & $2 k$ & 48 \\
\hline
\end{tabular}

${ }^{a}$ All reactions were performed with $0.5 \mathrm{mmol}$ of substrate, $0.5 \mathrm{mmol}$ of $\mathrm{H}_{2} \mathrm{O}, 0.25 \mathrm{mmol}$ of $\mathrm{CH}_{3} \mathrm{SO}_{3} \mathrm{H}$, and $5 \mathrm{~mol} \%$ of $\mathrm{Pt}(\mathrm{COD}) \mathrm{Cl}_{2}$ in $2.0 \mathrm{~mL}$ of $\mathrm{MeOH}$ at $70{ }^{\circ} \mathrm{C}$; ${ }^{b}$ Isolated yields; ${ }^{c} 1 \mathrm{~mL}$ of $\mathrm{MeOH}$ and $1 \mathrm{~mL}$ of $\mathrm{CH}_{2} \mathrm{Cl}_{2}$ were used as solvent.

Presumably, the mechanism in this reaction could be similar to that of the $\mathrm{PtCl}_{2}$-catalyzed hydrative cyclization of trialkyne functionalities [7]. We thus propose a mechanism (Scheme 1) involving an initial coordination of the diyne to $\mathrm{Pt}(\mathrm{II})$ to afford the intermediate $\mathbf{A}$. The addition of $\mathrm{H}_{2} \mathrm{O}$ takes place 
to form the $\alpha$-carbonyl platinum species $\mathbf{C}$. After a second hydration at the remaining alkyne of species $\mathbf{C}$, the resulting diketone species $\mathbf{D}$ undergoes a subsequent aldol condensation to form a product $\mathbf{2}$. Alternatively, cyclohexenone $\mathbf{2}$ could result from an alkyne insertion into intermediate $\mathbf{E}$, followed by hydrodemetalation of intermediate $\mathbf{F}$. The reason behind the catalytic activity of acid as an additive is unclear, although acid is proposed to exert a tuning effect on the activity of Pt catalysts.

Scheme 1. Proposed mechanism for The Pt-catalyzed hydrative cyclization of 1, 6-diynes.

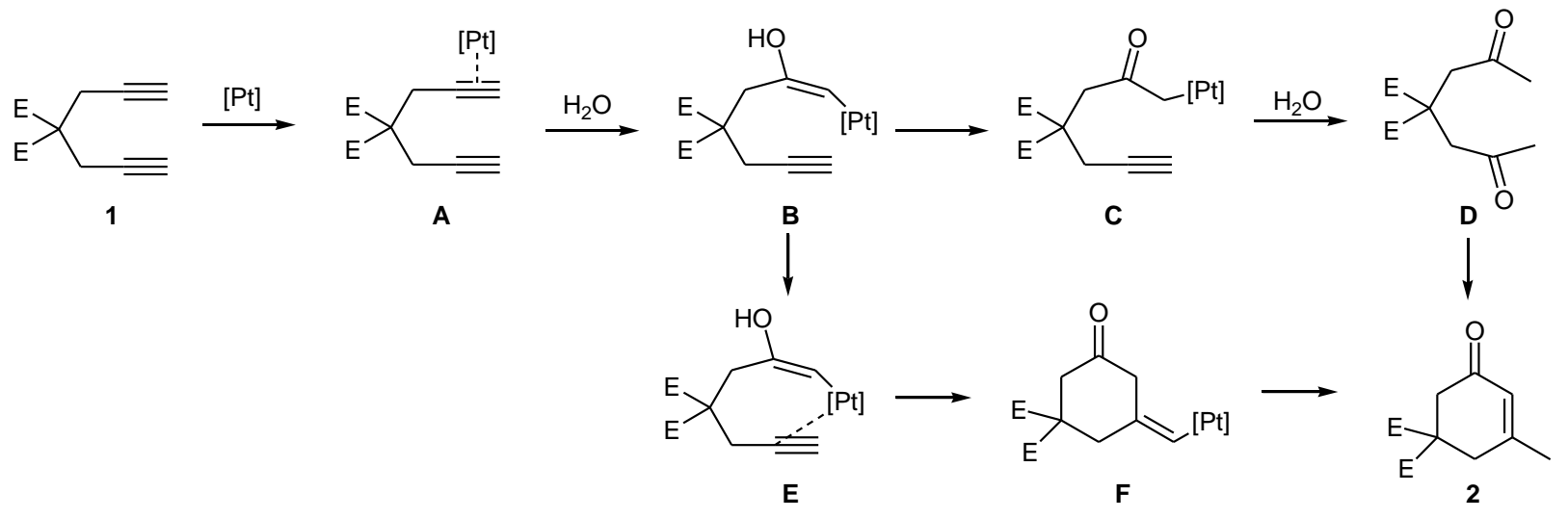

\section{Experimental}

\subsection{General}

Under otherwise noted, materials were obtained from commercial suppliers and used without further purification. Diynes were prepared by the procedures in the literature $[29,30]$. Thin layer chromatography (TLC) was performed using silica gel $60 \mathrm{~F}_{254}$ and visualized using UV light. Column chromatography was performed with silica gel (mesh 300-400). ${ }^{1} \mathrm{H}-\mathrm{NMR}$ and ${ }^{13} \mathrm{C}-\mathrm{NMR}$ spectra were recorded on a Bruker Avance $400 \mathrm{MHz}$ or $500 \mathrm{MHz}$ spectrometer in $\mathrm{CDCl}_{3}$ with $\mathrm{Me}_{4} \mathrm{Si}$ as an internal standard. Data are reported as follows: chemical shifts in ppm $(\delta)$, multiplicity ( $=$ singlet, $\mathrm{d}=$ doublet, $\mathrm{t}=$ triplet, $\mathrm{q}=$ quartet, $\mathrm{br}=$ broad and $\mathrm{m}=$ multiplet, coupling constant $(\mathrm{Hz})$ and integration. Infrared spectra (IR) were obtained on a 370 FT-IR spectrometer; absorptions are reported in $\mathrm{cm}^{-1}$. Mass spectra (MS) and high resolution mass spectra (HRMS) were obtained at the Zhejiang University of Technology Mass Spectrometry Facility.

\subsection{General procedure for the hydrative cyclization of diynes}

To a reactor containing diyne $(0.5 \mathrm{mmol})$, methanol $(2 \mathrm{~mL})$, and $\mathrm{H}_{2} \mathrm{O}(10 \mu \mathrm{L})$ under nitrogen $\mathrm{Pt}(\mathrm{COD}) \mathrm{Cl}_{2}(9.0 \mathrm{mg}, 0.025 \mathrm{mmol}, 5 \mathrm{~mol} \%)$ and $\mathrm{CH}_{3} \mathrm{SO}_{3} \mathrm{H}(20 \mu \mathrm{L})$ were added. The resulting yellow solution was then sealed and stirred at $70{ }^{\circ} \mathrm{C}$ for 3-13 hours until the starting diyne was consumed, as judged by TLC. The mixture was quenched with a saturated solution of $\mathrm{NaHCO}_{3}$ and then extracted with ethyl acetate $(20 \mathrm{~mL} \times 3)$. The organic layer was washed with brine, dried over $\mathrm{Na}_{2} \mathrm{SO}_{4}$ and concentrated in vacuo. The residue was purified by column chromatography (silica gel) (Eluent: hexane/ethyl acetate) to yield the corresponding cyclized product in an analytically pure form. 
Dimethyl 3-methyl-5-oxocyclohex-3-ene-1,1-dicarboxylate (2a) [24]. A pale yellow oil; ${ }^{1} \mathrm{H}-\mathrm{NMR}$ $\left(400 \mathrm{MHz} \mathrm{CDCl}_{3}\right) \delta 5.88(\mathrm{~s}, 1 \mathrm{H}), 3.75(\mathrm{~s}, 6 \mathrm{H}), 2.90(\mathrm{~s}, 2 \mathrm{H}), 2.87(\mathrm{~s}, 2 \mathrm{H}), 2.01(\mathrm{~s}, 3 \mathrm{H}) ;{ }^{13} \mathrm{C}-\mathrm{NMR}(100$ $\left.\mathrm{MHz}, \mathrm{CDCl}_{3}\right) \delta 194.5,170.2,158.7,126.2,55.5,53.3,41.7,36.3,24.3$.

Diethyl 3-methyl-5-oxocyclohex-3-ene-1,1-dicarboxylate (2b) [24]. A colorless oil; ${ }^{1} \mathrm{H}-\mathrm{NMR}$ $\left(400 \mathrm{MHz}, \mathrm{CDCl}_{3}\right) \delta 5.88(\mathrm{q}, J=1.2 \mathrm{~Hz}, 1 \mathrm{H}), 4.20(\mathrm{q}, J=7.0 \mathrm{~Hz}, 4 \mathrm{H}), 2.89(\mathrm{~s}, 2 \mathrm{H}), 2.86(\mathrm{~s}, 2 \mathrm{H}), 2.01$ $(\mathrm{d}, J=1.2 \mathrm{~Hz}, 3 \mathrm{H}), 1.24(\mathrm{t}, J=7.0 \mathrm{~Hz}, 6 \mathrm{H}) ;{ }^{13} \mathrm{C}-\mathrm{NMR}\left(100 \mathrm{MHz}, \mathrm{CDCl}_{3}\right) \delta 194.8,169.8,158.7$, $126.2,62.2,55.5,41.7,36.2,24.3,13.9$.

5,5-Bis(hydroxymethyl)-3-methylcyclohex-2-enone (2c) [25]. White solid, m.p.: 64-65 ${ }^{\circ} \mathrm{C} .{ }^{1} \mathrm{H}-\mathrm{NMR}$ $\left(500 \mathrm{MHz}, \mathrm{CDCl}_{3}\right) \delta 5.88(\mathrm{~s}, 1 \mathrm{H}), 3.91(\mathrm{br}, 2 \mathrm{H}), 3.55(\mathrm{~s}, 4 \mathrm{H}), 2.30(\mathrm{~s}, 4 \mathrm{H}), 1.98(\mathrm{~s}, 3 \mathrm{H}) ;{ }^{13} \mathrm{C}-\mathrm{NMR}$ $\left(125 \mathrm{MHz}, \mathrm{CDCl}_{3}\right) \delta 199.8,161.4,125.6,66.5,42.4,40.8,35.0,24.6$.

5-(Hydroxymethyl)-5-(methoxymethyl)-3-methylcyclohex-2-enone (2d). A pale yellow oil; ${ }^{1} \mathrm{H}-\mathrm{NMR}$ (400 MHz, $\left.\mathrm{CDCl}_{3}\right) \delta 5.84-5.83(\mathrm{~m}, 1 \mathrm{H}), 3.53-3.45$ (m, 2H), 3.35-3.26 (m, 5H), 2.88 (br, 1H), 2.36 (s, 2H), $2.24(\mathrm{~s}, 2 \mathrm{H}), 1.92(\mathrm{~s}, 3 \mathrm{H}) ;{ }^{13} \mathrm{C}-\mathrm{NMR}\left(100 \mathrm{MHz}, \mathrm{CDCl}_{3}\right) \delta$ 198.6, 160.2, 125.6, 77.5, 67.2, 59.4, 41.8, 41.2, 34.9, 24.2; IR (KBr) $v_{\max } 3445,2927,1651,1382,1104 \mathrm{~cm}^{-1}$; HRMS (EI) for $\mathrm{C}_{10} \mathrm{H}_{16} \mathrm{O}_{3}$ : calcd. 184.1099. Found 184.1097.

5,5-Bis(methoxymethyl)-3-methylcyclohex-2-enone (2e) [24]. A pale yellow oil; ${ }^{1} \mathrm{H}-\mathrm{NMR}$ (400 MHz, $\left.\mathrm{CDCl}_{3}\right) \delta 5.86(\mathrm{~s}, 1 \mathrm{H}), 3.31(\mathrm{~s}, 6 \mathrm{H}), 3.23(\mathrm{~s}, 4 \mathrm{H}), 2.34(\mathrm{~s}, 2 \mathrm{H}), 2.32(\mathrm{~s}, 2 \mathrm{H}), 1.94(\mathrm{~s}, 3 \mathrm{H}) ;{ }^{13} \mathrm{C}-\mathrm{NMR}$ $\left(100 \mathrm{MHz} \mathrm{CDCl}_{3}\right) \delta 198.9,159.8,125.4,75.4,59.2,41.6,41.3,34.8,24.3$.

Ethyl 1-(diphenylphosphoryl)-3-methyl-5-oxocyclohex-3-enecarboxylate (2f) [24]. White solid, m.p. 122.3-125.5 ${ }^{\circ} \mathrm{C} .{ }^{1} \mathrm{H}$ NMR $\left(400 \mathrm{MHz}, \mathrm{CDCl}_{3}\right) \delta$ 8.06-8.02 (m, 2H), 7.90-7.85 (m, 2H), 7.69-7.47 $(\mathrm{m}, 6 \mathrm{H}), 5.84(\mathrm{~s}, 1 \mathrm{H}), 3.93-3.79(\mathrm{~m}, 2 \mathrm{H}), 3.03-2.83(\mathrm{~m}, 4 \mathrm{H}), 1.92(\mathrm{~s}, 3 \mathrm{H}), 0.91-0.86(\mathrm{~m}, 3 \mathrm{H}) ;{ }^{13} \mathrm{C}-$ NMR $\left(100 \mathrm{MHz}, \mathrm{CDCl}_{3}\right) \delta 194.4(\mathrm{~d}, J \mathrm{c}-\mathrm{p}=11.3 \mathrm{~Hz}), 170.8(\mathrm{~d}, J \mathrm{c}-\mathrm{p}=20.2 \mathrm{~Hz}), 159.2(\mathrm{~d}, J \mathrm{c}-\mathrm{p}=$ $12.4 \mathrm{~Hz}), 132.2(\mathrm{q}, J \mathrm{c}-\mathrm{p}=2.8 \mathrm{~Hz}), 131.9(\mathrm{~d}, J \mathrm{c}-\mathrm{p}=8.9 \mathrm{~Hz}), 131.6(\mathrm{~d}, J \mathrm{c}-\mathrm{p}=8.9 \mathrm{~Hz}), 129.0$ $(\mathrm{d}, J \mathrm{c}-\mathrm{p}=10.6 \mathrm{~Hz}), 128.3(\mathrm{~d}, J \mathrm{c}-\mathrm{p}=2 \mathrm{~Hz}), 128.2(\mathrm{~d}, J \mathrm{c}-\mathrm{p}=2 \mathrm{~Hz}), 128.0(\mathrm{~d}, J \mathrm{c}-\mathrm{p}=11 \mathrm{~Hz}), 125.6$, 61.7, $53.0(\mathrm{~d}, J$ c-p $=57 \mathrm{~Hz}), 39.1,33.9,24.1,20.6(\mathrm{~d}, J$ c-p = 4.1 Hz), 13.1.

Methyl 3-methyl-5-oxocyclohex-3-enecarboxylate (2g) [24]. A pale yellow oil; ${ }^{1} \mathrm{H}-\mathrm{NMR}$ (400 MHz, $\left.\mathrm{CDCl}_{3}\right) \delta 5.91(\mathrm{~s}, 1 \mathrm{H}), 3.72(\mathrm{~s}, 3 \mathrm{H}), 3.10-3.04(\mathrm{~m}, 1 \mathrm{H}), 2.67-2.51(\mathrm{~m}, 4 \mathrm{H}), 2.00(\mathrm{~s}, 3 \mathrm{H}) ;{ }^{13} \mathrm{C}-\mathrm{NMR}$ $\left(100 \mathrm{MHz} \mathrm{CDCl}_{3}\right) \delta 196.8,173.5,160.2,126.5,52.1,39.6,38.6,33.0,24.2$.

Methyl 3-methyl-5-oxo-1-phenylcyclohex-3-enecarboxylate (2h) [24]. Colorless crystals; m.p. 83.0-84.0. ${ }^{1} \mathrm{H}-\mathrm{NMR}\left(400 \mathrm{MHz}, \mathrm{CDCl}_{3}\right) \delta$ 7.37-7.28 (m, 5H), 5.94-5.93 (m, 1H), $3.64(\mathrm{~s}, 3 \mathrm{H})$, 3.29-3.21 (m, 2H), 2.81-2.73 (m, 2H), 2.05 (s, 3H); ${ }^{13} \mathrm{C}-\mathrm{NMR}\left(100 \mathrm{MHz}, \mathrm{CDCl}_{3}\right) \delta$ 196.6, 174.0, $160.4,140.0,128.9,127.7,126.5,125.5,52.8,51.8,45.1,40.1,24.6$.

Methyl 1-acetyl-3-methyl-5-oxocyclohex-3-enecarboxylate (2i) [25]. A pale yellow oil; ${ }^{1} \mathrm{H}$ NMR $\left(400 \mathrm{MHz}, \mathrm{CDCl}_{3}\right) \delta 5.87(\mathrm{~s}, 1 \mathrm{H}), 3.76(\mathrm{~s}, 3 \mathrm{H}), 2.93(\mathrm{~d}, J=16.4 \mathrm{~Hz}, 1 \mathrm{H}), 2.84(\mathrm{~d}, J=0.8 \mathrm{~Hz}, 2 \mathrm{H})$, $2.72(\mathrm{~d}, J=16.4 \mathrm{~Hz}, 1 \mathrm{H}), 2.20(\mathrm{~s}, 3 \mathrm{H}), 2.01(\mathrm{~s}, 3 \mathrm{H}) ;{ }^{13} \mathrm{C} \mathrm{NMR}\left(100 \mathrm{MHz}, \mathrm{CDCl}_{3}\right) \delta 202.0,194.8$, $170.9,158.8,126.1,61.6,53.2,41.2,35.4,25.8,24.3$. 
Methyl 1-(1-hydroxyethyl)-3-methyl-5-oxocyclohex-3-enecarboxylate (2j) [25]. A pale yellow oil; ${ }^{1} \mathrm{H}$ NMR $\left(400 \mathrm{MHz}, \mathrm{CDCl}_{3}\right) \delta 5.85(\mathrm{~s}, 1 \mathrm{H}), 3.87-2.84(\mathrm{~m}, 1 \mathrm{H}), 3.69(\mathrm{~s}, 3 \mathrm{H}), 2.88-2.79(\mathrm{~m}, 2 \mathrm{H})$, 2.70-2.66 (br, 1H), 2.62-2.36 (m, 2H), 2.16-1.84 (m, 3H), 1.25-1.17 (m, 3H); ${ }^{13} \mathrm{C}-\mathrm{NMR}(100 \mathrm{MHz}$, $\left.\mathrm{CDCl}_{3}\right) \delta 197.5,174.7,160.4,126.0,71.5,54.0,52.4,41.6,35.5,24.5,18.7$.

5-Fluorene-3-methylcyclohex-2-enone (2k) [25]. A white solid; m.p. 167-168 ${ }^{\circ} \mathrm{C} ;{ }^{1} \mathrm{H}-\mathrm{NMR}(500 \mathrm{MHz}$, $\left.\mathrm{CDCl}_{3}\right) \delta 7.74(\mathrm{~d}, J=7.5 \mathrm{~Hz}, 2 \mathrm{H}), 7.48(\mathrm{~d}, J=7.5 \mathrm{~Hz}, 2 \mathrm{H}), 7.40-7.37(\mathrm{~m}, 2 \mathrm{H}), 7.29-7.26(\mathrm{~m}, 2 \mathrm{H}), 6.27$ $(\mathrm{d}, J=1 \mathrm{~Hz}, 1 \mathrm{H}), 2.68(\mathrm{~s}, 4 \mathrm{H}), 2.02(\mathrm{~s}, 3 \mathrm{H}) ;{ }^{13} \mathrm{C}-\mathrm{NMR}\left(125 \mathrm{MHz}, \mathrm{CDCl}_{3}\right) \delta 198.1,160.4,149.9$, $139.3,127.9,127.6,127.1,123.0,120.1,51.0,45.7,41.2,24.5$.

\section{Conclusions}

In summary, various 3,5-substituted conjugated cyclohexenones were synthesized by $\mathrm{Pt}$ (II)-catalyzed hydrative cyclization of 1,6-diynes. Advantages of the present method are the easily accessible starting materials, mild conditions, lack of coproducts and the fact that several types of functional groups were tolerated. Further studies are underway to expand the scope of the present method and are directed toward further method development on these cyclohexenone scaffolds as well as applications in natural product and the bioactive molecule synthesis.

\section{Acknowledgements}

This work was partially supported by the Natural Science Foundations of China (Nos. 20672099, 20572094).

\section{References and Notes}

1. Braca, A.; Tommasi, N.D.; Bari, L.-D.; Pizza, C.; Politi, M.; Morelli, I. Antioxidant principles from Bauhinia tarapotensis. J. Nat. Prod. 2001, 64, 892-895.

2. Girlanda-Junges, C.; Keyling-Bilger, F.; Schmitt, G.; Luu, B. Effect of cyclohexenonic long chain fatty alcohols on neurite outgrowth. Study on structure-activity relationship. Tetrahedron 1998, 54, 7735-7748.

3. Ho, T.-L. Symmetry: A Basis for Synthesis Design; Wiley: New York, NY, USA, 1995; pp. 290-348.

4. Corey, E.J.; Cheng, X.-M. The Logic of Chemical Synthesis; Wiley: New York, NY, USA, 1989.

5. Zhang, F.Y.; Corey, E.J. Highly Enantioselective Michael Reactions Catalyzed by a Chiral Quaternary Ammonium Salt. Illustration by Asymmetric Syntheses of (S)-Ornithine and Chiral 2Cyclohexenones. Org. Lett. 2000, 2, 1097-1100.

6. Hareau, G.P.-J.; Koiwa, M.; Hikichi, S.; Sato, F. Synthesis of Optically Active 5-(tertButyldimethylsiloxy)-2-cyclohexenone and Its 6-Substituted Derivatives as Useful Chiral Building Blocks for the Synthesis of Cyclohexane Rings. Syntheses of Carvone, Penienone, and Penihydrone. J. Am. Chem. Soc. 1999, 121, 3640-3650.

7. Chong, B.-D.; Ji, Y.-I.; Oh, S.-S.; Yang, J.-D.; Baik, W.; Koo, S. Highly Efficient Synthesis of Methyl-Substituted Conjugate Cyclohexenones. J. Org. Chem. 1997, 62, 9323-9325. 
8. Kuehne, M.E.; Parsons, W.H. Thiyl radical- and mercuric ion-induced cyclizations of dimethyl dipropargylmalonate and dimethyl propargyl-3-thiylallylmalonates. J. Org. Chem. 1977, 42, 3408-3410.

9. Phillips, D.D.; Johnson, A.W. Notes - A Novel Synthesis of Cyclohexenones by Intramolecular Acylation. J. Org. Chem. 1956, 21, 587-589.

10. Dauben, W.G.; Michno, D.M. Direct oxidation of tertiary allylic alcohols. A simple and effective method for alkylative carbonyl transposition. J. Org. Chem. 1977, 42, 682-685.

11. Stork, G.; Danheiser, R.L.; Ganem, B. Reaction of deuterium chloride with cis- and trans-.beta.trimethylsilylstyrene. J. Am. Chem. Soc. 1973, 95, 3414-3415.

12. Stotter, P.L.; Hill, K.A. alpha.-Halocarbonyl compounds. II. Position-specific preparation of .alpha.-bromoketones by bromination of lithium enolates. Position-specific introduction of .alpha.,.beta.-unsaturation into unsymmetrical ketones. J. Org. Chem. 1973, 38, 2576-2578.

13. Rubinstein, H. Intermediates in the Synthesis of Xanthophyll and Zeaxanthin. J. Org. Chem. 1962, 27, 3886-3887.

14. Trost, B.M.; Rudd, M.T. Ruthenium-Catalyzed Cycloisomerizations of Diynols. J. Am. Chem. Soc. 2005, 127, 4763-4776.

15. Odedra, A.; Wu, C.-J.; Pratap, T.B.; Huang, C.-W.; Ran, Y.F.; Liu, R.-S. Ruthenium-Catalyzed Aromatization of Enediynes via Highly Regioselective Nucleophilic Additions on a $\pi$-Alkyne Functionality. A Useful Method for the Synthesis of Functionalized Benzene Derivatives. J. Am. Chem. Soc. 2005, 127, 3406-3412.

16. Trost, B.M.; Huang, X.-J. Ruthenium-Catalyzed Diyne Hydrative Cyclization: Synthesis of Substituted 1,3-Diene Synthons. Org. Lett. 2005, 7, 2097-2099.

17. Trost, B.M.; Rudd, M.T. A Mechanistic Dichotomy in Ruthenium-Catalyzed Propargyl Alcohol Reactivity: A Novel Hydrative Diyne Cyclization. J. Am. Chem. Soc. 2003, 125, 11516-11517.

18. Trost, B.M.; Brown, R.E.; Toste, F.D. A Ruthenium-Catalyzed Hydrative Cyclization and [4 + 2] Cycloaddition of Yne-enones. J. Am. Chem. Soc. 2000, 122, 5877-5878.

19. Chen, Y.; Ho, D.M.; Lee, C. Ruthenium-Catalyzed Hydrative Cyclization of 1,5-Enynes. J. Am. Chem. Soc. 2005, 127, 12184-12185.

20. Trost, B.M.; Rudd, M.T. Ruthenium-Catalyzed Cycloisomerizations of Diynols. J. Am. Chem. Soc. 2005, 127, 4763-4776.

21. Chang, H.-K.; Datta, S.; Das, A.; Odedra, A.; Liu, R.-S. $\mathrm{PtCl}_{2}$-Catalyzed Hydrative Cyclization of Trialkyne Functionalities to Form Bicyclic Spiro Ketones. Angew. Chem. Int. Ed. 2007, 46, 4744-4747.

22. Chang, H.-K.; Liao, Y.-C.; Liu, R.-S. Diversity in Platinum-Catalyzed Hydrative Cyclization of Trialkyne Substrates To Form Tetracyclic Ketones. J. Org. Chem. 2007, 72, 8139-8141.

23. Baidossi, W.; Lahav, M.; Blum, J. Hydration of Alkynes by a $\mathrm{PtCl}_{4}-\mathrm{CO}$ Catalyst. J. Org. Chem. 1997, 62, 669-672.

24. Zhang, C.; Cui, D.-M.; Yao, L.-Y.; Wang, B.-S.; Hu, Y.-Z.; Hayashi, T. Synthesis of 2Cyclohexenone Derivatives via Gold(I)-Catalyzed Hydrative Cyclization of 1,6-Diynes. J. Org. Chem. 2008, 73, 7811-7813.

25. Cui, D.-M.; Ke, Y.-N.; Zhuang, D.-W.; Wang, Q.; Zhang, C. Gold-catalyzed hydrative cyclization of 1,6-diynes in ionic liquid media. Tetrahedron Lett. 2010, 51, 980-982. 
26. Oh, C.H.; Yi, H.J.; Lee, J.H. Silver-catalyzed intramolecular oxycyclization of alkynes to bridged bicyclic ketalsElectronic supplementary information (ESI) available: Characterization of new compounds 2a-d, 2f-i, 3a, 4b and 5b. New J. Chem. 2007, 31, 835-837;

27. Antoniotti, S.; Genin, E.; Michelet, V.; Genêt, J.-P. Highly Efficient Access to Strained Bicyclic Ketals via Gold-Catalyzed Cycloisomerization of Bis-homopropargylic Diols. J. Am. Chem. Soc. 2005, 127, 9976-9977.

28. Kinney, W.A.; Crouse, G.D.; Paquette, L.A. Regiocontrolled synthesis of mono-, di-, and trisubstituted cyclohexenones by cycloaddition of vinyl sulfones to 1-methoxy-3[(trimethylsilyl)oxy]-1,3-butadienes. Conversion of alkenes into effective dienophilic reagent. $J$. Org. Chem. 1983, 48, 4986.

29. Fox, H.; Wolf, M.O.; O’Dell, R.; Lin, B.L.; Schrock, R.R.; Wrighton, M.S. Living Cyclopolymerization of 1,6-Heptadiyne Derivatives Using Well-Defined Alkylidene Complexes: Polymerization Mechanism, Polymer Structure, and Polymer Properties. J. Am. Chem. Soc. 1994, 116, 2827.

30. Madine, J.W.; Wang, X.; Widenhoefer, R.A. Cyclization/Hydrosilylation of Functionalized Diynes Catalyzed by a Cationic Platinum Phenanthroline Complex. Org. Lett. 2001, 3, 385.

Sample Availability: Samples of the compounds are available from the authors.

(C) 2010 by the authors; licensee MDPI, Basel, Switzerland. This article is an Open Access article distributed under the terms and conditions of the Creative Commons Attribution license (http://creativecommons.org/licenses/by/3.0/). 